Article

\title{
Several Fixed Point Theorems in Convex $b$-Metric Spaces and Applications
}

\author{
Lili Chen ${ }^{1,2}$, Chaobo Li $^{2}$, Radoslaw Kaczmarek ${ }^{3}$ and Yanfeng Zhao ${ }^{1, *}$ \\ 1 College of Mathematics and Systems Science, Shandong University of Science and Technology, \\ Qingdao 266590, China; cll2119@hotmail.com \\ 2 Department of Mathematics, Harbin University of Science and Technology, Harbin 150080, China; \\ libo566588@126.com \\ 3 Poznań, Faculty of Mathematics and Computer Science, Adam Mickiewicz University, Uniwersytetu \\ Poznańskiego 4, 61-614 Poznań, Poland; radekk@amu.edu.pl \\ * Correspondence: zhaoyanfeng1101@126.com
}

Received: 3 January 2020; Accepted: 10 February 2020; Published: 14 February 2020

\begin{abstract}
Our paper is devoted to indicating a way of generalizing Mann's iteration algorithm and a series of fixed point results in the framework of $b$-metric spaces. First, the concept of a convex $b$-metric space by means of a convex structure is introduced and Mann's iteration algorithm is extended to this space. Next, by the help of Mann's iteration scheme, strong convergence theorems for two types of contraction mappings in convex $b$-metric spaces are obtained. Some examples supporting our main results are also presented. Moreover, the problem of the T-stability of Mann's iteration procedure for the above mappings in complete convex $b$-metric spaces is considered. As an application, we apply our main result to approximating the solution of the Fredholm linear integral equation.
\end{abstract}

Keywords: $b$-metric space; Mann's iteration scheme; fixed point theorems; convex structure

MSC: 47H09; 47H10

\section{Introduction}

In the last few decades one could observe a huge amount of interest for the development of the fixed point theory because of plenty of applications, especially in metric spaces [1,2]. Banach's contraction principle [3] is one of the most widely applied fixed point theorems in all branches of mathematics [4-12]. In recent decades, scholars have devoted themselves to extending the above theorem to all kinds of generalized metric spaces [13-17]. In 1993, Czerwik [18] introduced the concept of $b$-metric spaces by weakening the coefficient of the triangle inequality and generalized Banach's contraction principle to these spaces. Subsequently, Boriceanu, Bota and Petrusel $[19,20]$ obtained some concrete examples of $b$-metric spaces, and studied the fixed point properties of set-valued operators in $b$-metric spaces. The fixed point properties of $b$-metric spaces have received much attention; for example, see [21-28] and references therein.

In 1970, Takahashi [29] introduced the concepts of a convex structure and a convex metric space, and formulated some first fixed point theorems for nonexpansive mappings in the convex metric space. In addition, Goebel and Kirk [30] studied some iterative processes for nonexpansive mappings in the hyperbolic metric space, and in 1988, Xie [31] found fixed points of quasi-contraction mappings in convex metric spaces by Ishikawa's iteration scheme. In 1990, Reich and Shafrir [32] presented nonexpansive iterations in hyperbolic spaces. In general, the Picard iteration algorithm is widely used in studying the fixed point problems for many kinds of contraction mappings and quasi-contraction mappings in $b$-metric spaces. However, it is hard to extend other algorithms directly to the $b$-metric spaces because of the characteristics of this kind of metric space. 
In this work, we firstly introduce the concept of the convex $b$-metric space by means of the convex structure. Moreover, we extend Mann's iteration algorithm to the above space. We also present some specific examples of convex $b$-metric spaces. Furthermore, by means of Mann's iteration scheme, we obtain strong convergence theorems for two types of contraction mapping in convex $b$-metric spaces. In addition, we show concrete examples supporting our main results. Moreover, we introduce the concept of weak $T$-stability of the iteration for mappings in complete metric spaces and discuss the problem of weak T-stability of Mann's iteration procedure for above two kinds of mappings in complete convex $b$-metric spaces. As an application, we apply our main result to approximating the solution of the Fredholm linear integral equation.

\section{Preliminaries}

Firstly, we recall some basic notations of $b$-metric spaces.

Definition 1 ([21]). Let $H \neq \varnothing$ be a set and $s \geq 1$ be a given real number. A function $d_{b}: H \times H \rightarrow[0, \infty]$ is called a b-metric if the following hold, for every $u, v, o \in H$ :

(1) $d_{b}(u, v)=0$ if and only if $u=v$;

(2) $\quad d_{b}(u, v)=d_{b}(v, u)$;

(3) $\quad d_{b}(u, v) \leq s\left[d_{b}(u, o)+d_{b}(o, v)\right]$.

Then, the pair $\left(H, d_{b}\right)$ is called a b-metric space with constant $s \geq 1$.

Definition 2 ([18,32]). Let $\left\{u_{n}\right\}$ be a sequence in a b-metric space $\left(H, d_{b}\right)$. Then:

(1) The sequence $\left\{u_{n}\right\}$ is said to be convergent in $\left(H, d_{b}\right)$ if there exists $u^{*} \in H$ such that $\lim _{n \rightarrow \infty} d_{b}\left(u_{n}, u^{*}\right)=0$.

(2) The sequence $\left\{u_{n}\right\}$ is said to be a Cauchy sequence in $\left(H, d_{b}\right)$, if for every $\varepsilon>0$ there exists a positive $n_{0} \in \mathbb{N}$ such that $d_{b}\left(u_{n}, u_{m}\right)<\varepsilon$ for all $n, m>n_{0}$ (or, equivalently, $\lim _{n, m \rightarrow \infty} d_{b}\left(u_{n}, u_{m}\right)=0$ ).

(3) $\left(H, d_{b}\right)$ is called a complete b-metric space if every Cauchy sequence is convergent in $H$.

Definition 3 ([31]). Let $H \neq \varnothing$ and $I=[0,1]$. Define the mapping $d_{b}: H \times H \rightarrow[0, \infty]$ and a continuous function $w: H \times H \times I \rightarrow H$. Then $w$ is said to be the convex structure on $H$ if the following holds:

$$
d_{b}(o, w(u, v ; \lambda)) \leq \lambda d_{b}(o, u)+(1-\lambda) d_{b}(o, v)
$$

for each $o \in H$ and $(u, v ; \lambda) \in H \times H \times I$.

\section{Main Results}

In this section, we begin with the definition of a convex $b$-metric space.

Definition 4. Let the mapping $w: H \times H \times I \rightarrow H$ be a convex structure on a b-metric space $\left(H, d_{b}\right)$ with constant $s \geq 1$ and $I=[0,1]$. Then $\left(H, d_{b}, w\right)$ is said to be a convex b-metric space.

Let $\left(X, d_{b}, w\right)$ be a convex $b$-metric space and $T: X \rightarrow X$ be a mapping. We generalize Mann's iteration scheme to the convex $b$-metric space as follows:

$$
x_{n+1}=w\left(x_{n}, T x_{n} ; \alpha_{n}\right), n \in \mathbb{N},
$$

where $x_{n} \in X$ and $\alpha_{n} \in[0,1]$. The sequence $\left\{x_{n}\right\}$ is said to be Mann's iteration sequence for $T$.

Let us present now some specific examples of convex $b$-metric spaces.

Example 1. Let $H=\mathbb{R}$, and for any $u, v \in H$, let us define the metric $d_{b}: H \times H \rightarrow[0,+\infty)$ by the formula

$$
d_{b}(u, v)=|u-v|^{l}, l>1
$$


, and the mapping $w: H \times H \times\left\{\frac{1}{2}\right\} \rightarrow H$ by the formula

$$
w(u, v ; \alpha)=\frac{u+v}{2}
$$

Then, $\left(H, d_{b}, w\right)$ is a convex b-metric space with $s=2^{l-1}$. However, $\left(H, d_{b}, w\right)$ is not a metric space in the usual sense.

Indeed, by the help of the fact that for any $n, m \in[0,+\infty)$ and $l \geq 1$, inequality

$$
(n+m)^{l} \leq 2^{l-1}\left(n^{l}+m^{l}\right)
$$

holds, we easily show that $\left(H, d_{b}\right)$ is a b-metric space with $s=2^{l-1}$. Next we verify that w satisfies inequality (1). For any $0, u, v \in H$, we get

$$
\begin{aligned}
d_{b}(o, w(u, v ; \alpha)) & =\left|o-\left[\frac{u+v}{2}\right]\right|^{l} \\
& \leq 2^{l-1}\left[2^{-l}|o-u|^{l}+2^{-l}|o-v|^{l}\right] \\
& =2^{-1}\left[|o-u|^{l}+|o-v|^{l}\right] \\
& =\alpha d_{b}(o, u)+(1-\alpha) d_{b}(o, v)
\end{aligned}
$$

so $\left(H, d_{b}, w\right)$ is a convex $b$-metric space with $s=2^{l-1}$. However, $\left(H, d_{b}, w\right)$ is not a metric space in the usual sense because $d_{b}$ does not satisfy the classical triangle inequality. Indeed, if we put $l=2$, then

$$
d_{b}(2,4)=4>d_{b}(2,3)+d_{b}(3,4)=2
$$

Example 2. Let $H=\mathbb{R}^{n}$ and $d_{b}(u, v)=\sum_{i=1}^{n}\left(u_{i}-v_{i}\right)^{2}$ for all $u=\left(u_{1}, u_{2}, \cdots, u_{n}\right) \in H$ and $v=$ $\left(v_{1}, v_{2}, \cdots, v_{n}\right) \in H$. Obviously, $\left(H, d_{b}\right)$ is a b-metric space with $s=2$. Let $w: H \times H \times[0,1] \rightarrow H$ be the mapping defined as

$$
w(u, v ; \alpha)=\alpha u+(1-\alpha) v,
$$

for any $u, v \in H$. Then, $w$ satisfies inequality (1). Indeed, for all $o, u, v \in H$,

$$
\begin{aligned}
d_{b}(o, w(u, v ; \alpha))= & \sum_{i=1}^{n}\left(o_{i}-\left(\alpha u_{i}+(1-\alpha) v_{i}\right)\right)^{2} \\
\leq & \sum_{i=1}^{n}\left(\alpha\left|o_{i}-u_{i}\right|+(1-\alpha)\left|o_{i}-v_{i}\right|\right)^{2} \\
= & \sum_{i=1}^{n} \alpha^{2}\left(o_{i}-u_{i}\right)^{2}+\sum_{i=1}^{n}(1-\alpha)^{2}\left(o_{i}-v_{i}\right)^{2} \\
& +2 \alpha(1-\alpha) \sum_{i=1}^{n}\left|o_{i}-u_{i}\right| \cdot\left|o_{i}-v_{i}\right| \\
\leq & \sum_{i=1}^{n} \alpha^{2}\left(o_{i}-u_{i}\right)^{2}+\sum_{i=1}^{n}(1-\alpha)^{2}\left(o_{i}-v_{i}\right)^{2} \\
& +\alpha(1-\alpha) \sum_{i=1}^{n}\left(\left(o_{i}-u_{i}\right)^{2}+\left(o_{i}-v_{i}\right)^{2}\right) \\
= & \alpha d_{b}(o, u)+(1-\alpha) d_{b}(o, v) .
\end{aligned}
$$

Hence, $\left(H, d_{b}, w\right)$ is a convex b-metric space. However, similarly to Example 1 , it is not a metric space in the usual sense. 
The next example shows that the mapping $w$ defined in Example 2, sometimes may not be a convex structure on some metric spaces (see $[33,34])$.

Example 3. Let $H=l^{p}=\left\{\left\{u_{n}\right\} \subset \mathbb{R}: \sum_{n=1}^{\infty}\left|u_{n}\right|^{p}<\infty\right\}$, where $0<p<1$. We define $d_{b}: H \times H \rightarrow$ $[0,+\infty)$ by the formula

$$
d_{b}(u, v)=\left(\sum_{n=1}^{\infty}\left|u_{n}-v_{n}\right|^{p}\right)^{1 / p}, u=\left\{u_{n}\right\}_{n=1}^{\infty}, v=\left\{v_{n}\right\}_{n=1}^{\infty} \in l^{p}
$$

Applying inequality $(n+m)^{p} \leq n^{p}+m^{p}(0<p<1)$ holding for all $n, m \in[0,+\infty)$, we easily obtain that $\left(H, d_{b}\right)$ is a b-metric space with constant $s=2^{(1-p) / p}$. Let $w: H \times H \times[0,1] \rightarrow H$ be the mapping defined as

$$
w(u, v ; \alpha)=\alpha u+(1-\alpha) v, \text { for all } u, v \in H .
$$

Assume that $u_{n}<o_{n}$ and $v_{n}<o_{n}$ for any $n \in \mathbb{N}$. Then, for all $\alpha \in(0,1)$, we get

$$
\begin{aligned}
d_{b}(o, w(u, v ; \alpha)) & =\left(\sum_{n=1}^{\infty}\left|\alpha\left(o_{n}-u_{n}\right)+(1-\alpha)\left(o_{n}-v_{n}\right)\right|^{p}\right)^{1 / p} \\
& >\left(\sum_{n=1}^{\infty}\left[\alpha\left(o_{n}-u_{n}\right)\right]^{p}\right)^{1 / p}+\left(\sum_{n=1}^{\infty}\left[(1-\alpha)\left(o_{n}-v_{n}\right)\right]^{p}\right)^{1 / p} \\
& =\alpha d_{b}(o, u)+(1-\alpha) d_{b}(o, v),
\end{aligned}
$$

which implies that $w$ is not a convex structure on $H$.

Now we will prove Banach's contraction principle for complete convex $b$-metric spaces by means of Mann's iteration algorithm.

Theorem 1. Let $\left(H, d_{b}, w\right)$ be a complete convex b-metric space with constant $s>1$ and $T: H \rightarrow H$ be a contraction mapping; that is, there exists $\beta \in[0,1)$ such that

$$
d_{b}(T u, T v) \leq \beta d_{b}(u, v), \text { for all } u, v \in H
$$

Let us choose $u_{0} \in H$ in such a way that $d_{b}\left(u_{0}, T u_{0}\right)=M<\infty$ and define $u_{n}=w\left(u_{n-1}, T u_{n-1} ; \alpha_{n-1}\right)$, where $0 \leq \alpha_{n-1}<1$ and $n \in \mathbb{N}$. If $\beta s^{4}<1$ and $0<\alpha_{n-1}<\frac{\frac{1}{s^{4}}-\beta}{1-\beta}$ for each $n \in \mathbb{N}$; then, $T$ has a unique fixed point in $H$.

Proof. Note that for any $n \in \mathbb{N}$, there holds

$$
d_{b}\left(u_{n}, u_{n+1}\right)=d_{b}\left(u_{n}, w\left(u_{n}, T u_{n} ; \alpha_{n}\right)\right) \leq\left(1-\alpha_{n}\right) d_{b}\left(u_{n}, T u_{n}\right)
$$

and

$$
\begin{aligned}
d_{b}\left(u_{n}, T u_{n}\right) & \leq s d_{b}\left(u_{n}, T u_{n-1}\right)+s d_{b}\left(T u_{n-1}, T u_{n}\right) \\
& \leq s d_{b}\left(w\left(u_{n-1}, T u_{n-1} ; \alpha_{n-1}\right), T u_{n-1}\right)+s \beta d_{b}\left(u_{n-1}, u_{n}\right) \\
& \leq s\left[\alpha_{n-1} d_{b}\left(u_{n-1}, T u_{n-1}\right)+\beta\left(1-\alpha_{n-1}\right) d_{b}\left(u_{n-1}, T u_{n-1}\right)\right] \\
& =s\left[\alpha_{n-1}+\beta\left(1-\alpha_{n-1}\right)\right] d_{b}\left(u_{n-1}, T u_{n-1}\right)
\end{aligned}
$$


Let $\lambda_{n-1}=s\left[\alpha_{n-1}+\beta\left(1-\alpha_{n-1}\right)\right]$. Combining this and the above inequality with assumptions $\beta s^{4}<1$ and $0<\alpha_{n-1}<\frac{\frac{1}{s^{4}}-\beta}{1-\beta}$ holding for each $n \in \mathbb{N}$, we get

$$
d_{b}\left(u_{n}, T u_{n}\right) \leq \lambda_{n-1} d_{b}\left(u_{n-1}, T u_{n-1}\right)<\frac{1}{s^{3}} d_{b}\left(u_{n-1}, T u_{n-1}\right)
$$

which implies that $\left\{d_{b}\left(u_{n}, T u_{n}\right)\right\}$ is a decreasing sequence of non-negative reals. Hence, there exists $\gamma \geq 0$ such that

$$
\lim _{n \rightarrow \infty} d_{b}\left(u_{n}, T u_{n}\right)=\gamma .
$$

We will show that $\gamma=0$. Suppose that $\gamma>0$. Letting $n \rightarrow \infty$ in inequality (2), we obtain

$$
\gamma \leq \frac{1}{s^{3}} \cdot \gamma<\gamma
$$

a contradiction. Hence, we get that $\gamma=0$. Moreover, we have

$$
d_{b}\left(u_{n}, u_{n+1}\right) \leq\left(1-\alpha_{n}\right) d_{b}\left(u_{n}, T u_{n}\right)<d_{b}\left(u_{n}, T u_{n}\right),
$$

which shows that $\lim _{n \rightarrow \infty} d_{b}\left(u_{n}, u_{n+1}\right)=0$. Now we will show that $\left\{u_{n}\right\}$ is a Cauchy sequence. Indeed, if $\left\{u_{n}\right\}$ is not a Cauchy sequence, then there exist $\varepsilon_{0}>0$ and the subsequences $\left\{u_{\theta(k)}\right\}$ and $\left\{u_{\eta(k)}\right\}$ of $\left\{u_{n}\right\}$, such that $\theta(k)$ is the smallest natural index with $\theta(k)>\eta(k)>k$,

$$
d_{b}\left(u_{\theta(k)}, u_{\eta(k)}\right) \geq \varepsilon_{0}
$$

and

$$
d_{b}\left(u_{\theta(k)-1}, u_{\eta(k)}\right)<\varepsilon_{0}
$$

Then, we conclude

$$
\varepsilon_{0} \leq d_{b}\left(u_{\theta(k)}, u_{\eta(k)}\right) \leq s\left[d_{b}\left(u_{\theta(k)}, u_{\eta(k)+1}\right)+d_{b}\left(u_{\eta(k)+1}, u_{\eta(k)}\right)\right],
$$

which implies that

$$
\frac{\varepsilon_{0}}{s} \leq \limsup _{k \rightarrow \infty} d_{b}\left(u_{\theta(k)}, u_{\eta(k)+1}\right)
$$

Noticing that

$$
\begin{aligned}
d_{b}\left(u_{\theta(k)}, u_{\eta(k)+1}\right)= & d_{b}\left(w\left(u_{\theta(k)-1}, T u_{\theta(k)-1} ; \alpha_{\theta(k)-1}\right), u_{\eta(k)+1}\right) \\
\leq & \alpha_{\theta(k)-1} d_{b}\left(u_{\theta(k)-1}, u_{\eta(k)+1}\right)+\left(1-\alpha_{\theta(k)-1}\right) d_{b}\left(T u_{\theta(k)-1}, u_{\eta(k)+1}\right) \\
\leq & \alpha_{\theta(k)-1} d_{b}\left(u_{\theta(k)-1}, u_{\eta(k)+1}\right)+\left(1-\alpha_{\theta(k)-1}\right) s\left[d_{b}\left(T u_{\theta(k)-1}, T u_{\eta(k)+1}\right)\right. \\
& \left.+d_{b}\left(T u_{\eta(k)+1}, u_{\eta(k)+1}\right)\right] \\
\leq & \alpha_{\theta(k)-1} d_{b}\left(u_{\theta(k)-1}, u_{\eta(k)+1}\right)+\left(1-\alpha_{\theta(k)-1}\right) s\left[\beta d_{b}\left(u_{\theta(k)-1}, u_{\eta(k)+1}\right)\right. \\
& \left.+d_{b}\left(T u_{\eta(k)+1}, u_{\eta(k)+1}\right)\right] \\
= & {\left[\alpha_{\theta(k)-1}+\left(1-\alpha_{\theta(k)-1}\right) s \beta\right] d_{b}\left(u_{\theta(k)-1}, u_{\eta(k)+1}\right) } \\
& +\left(1-\alpha_{\theta(k)-1}\right) s d_{b}\left(T u_{\eta(k)+1}, u_{\eta(k)+1}\right) \\
< & s\left[\alpha_{\theta(k)-1} s+\left(1-\alpha_{\theta(k)-1}\right) s \beta\right]\left(d_{b}\left(u_{\theta(k)-1}, u_{\eta(k)}\right)+d_{b}\left(u_{\eta(k)}, u_{\eta(k)+1}\right)\right) \\
& +\left(1-\alpha_{\theta(k)-1}\right) s d_{b}\left(T u_{\eta(k)+1}, u_{\eta(k)+1}\right),
\end{aligned}
$$


we obtain

$$
\frac{1}{s} \varepsilon_{0} \leq \limsup _{k \rightarrow \infty} d_{b}\left(u_{\theta(k)}, u_{\eta(k)+1}\right) \leq s^{2} \cdot \frac{1}{s^{4}} \varepsilon_{0}<\frac{1}{s} \varepsilon_{0},
$$

a contradiction. Thus $\left\{u_{n}\right\}$ is a Cauchy sequence in $H$. By the completeness of $H$, there exists $u^{*} \in H$ such that $\lim _{n \rightarrow \infty} d_{b}\left(u_{n}, u^{*}\right)=0$.

Next, we will verify that $u^{*}$ is a fixed point of $T$. Note that

$$
\begin{aligned}
d_{b}\left(u^{*}, T u^{*}\right) & \leq s\left[d_{b}\left(u^{*}, u_{n}\right)+d_{b}\left(u_{n}, T u^{*}\right)\right] \\
& \leq s d_{b}\left(u^{*}, u_{n}\right)+s^{2}\left[d_{b}\left(u_{n}, T u_{n}\right)+d_{b}\left(T u_{n}, T u^{*}\right)\right] \\
& =s d_{b}\left(u^{*}, u_{n}\right)+s^{2} d_{b}\left(u_{n}, T u_{n}\right)+s^{2} \beta d_{b}\left(u_{n}, u^{*}\right)
\end{aligned}
$$

Letting $n \rightarrow \infty$, we deduce that $d_{b}\left(u^{*}, T u^{*}\right)=0$ which implies that $T u^{*}=u^{*}$. Hence, $u^{*}$ is a fixed point of $T$. Now we will explain that $T$ has a unique fixed point. Suppose that $q \in H$ is another fixed point, that is, $T q=q$. Then,

$$
d_{b}\left(u^{*}, q\right)=d_{b}\left(T u^{*}, T q\right) \leq \beta d_{b}\left(u^{*}, q\right)
$$

for some $\beta \in[0,1)$, a contradiction. Hence, $u^{*}=q$ which completes the proof.

Let us give an example illustrating the above theorem.

Example 4. Let $H=\mathbb{R}^{+} \cup\{0\}$ and $T u=\frac{u}{5}$ for all $u \in H$. For any $u, v \in H$, we define function $d_{b}: H \times H \rightarrow[0,+\infty)$ by the formula $d_{b}(u, v)=(u-v)^{2}$, while the mapping $w: H \times H \times[0,1] \rightarrow H$ is defined as

$$
w(u, v ; \alpha)=\alpha u+(1-\alpha) v, \text { for all } u, v \in H .
$$

Set $\beta=\frac{1}{1+2^{4}}$ and $u_{n}=w\left(u_{n-1}, T u_{n-1} ; \alpha_{n-1}\right)$, where $u_{0}=1$ and $\alpha_{n-1}=\frac{1}{2^{4}}-\beta$. Then, $\left(H, d_{b}, w\right)$ is a complete convex b-metric space with $s=2$, and $T$ has a unique fixed point in $H$.

Indeed, from Example 1 it follows that $\left(H, d_{b}\right)$ is a b-metric space with $s=2$. In addition, for any $o, u, v \in H$, we have

$$
\begin{aligned}
d_{b}(o, w(u, v ; \alpha)) & =[\alpha(o-u)+(1-\alpha)(o-v)]^{2} \\
& \leq[\alpha|o-u|+(1-\alpha)|o-v|]^{2} \\
& =(\alpha|o-u|)^{2}+((1-\alpha)|o-v|)^{2}+2 \alpha(1-\alpha)|o-u||o-v| \\
& \leq(\alpha|o-u|)^{2}+((1-\alpha)|o-v|)^{2}+\alpha(1-\alpha)\left(|o-u|^{2}+|o-v|^{2}\right) \\
& =\alpha(o-u)^{2}+(1-\alpha)(o-v)^{2} \\
& =\alpha d_{b}(o, u)+(1-\alpha) d_{b}(o, v) .
\end{aligned}
$$

Hence, $\left(H, d_{b}, w\right)$ is a convex b-metric space with $s=2$. It is not difficult to see that $T$ satisfies

$$
d_{b}(T u, T v)=\frac{1}{25} d_{b}(u, v) \leq \beta d_{b}(u, v)
$$

where $\beta=\frac{1}{17}$. We choose $u_{0} \in H \backslash\{0\}$. Combining with $u_{n}=w\left(u_{n-1}, T u_{n-1} ; \alpha_{n-1}\right)$ and $\mathrm{Tu}=\frac{u}{5}$, we have

$$
u_{n}=\alpha_{n-1} u_{n-1}+\left(1-\alpha_{n-1}\right) T u_{n-1}=\left(\frac{1}{5}+\frac{4}{5} \alpha_{n-1}\right) u_{n-1}
$$

and

$$
u_{n-1}=\left(\frac{1}{5}+\frac{4}{5} \alpha_{n-2}\right) u_{n-2}, u_{n-2}=\left(\frac{1}{5}+\frac{4}{5} \alpha_{n-3}\right) u_{n-3}, \cdots, u_{1}=\left(\frac{1}{5}+\frac{4}{5} \alpha_{0}\right) u_{0} .
$$


Since $\alpha_{n-1}=\frac{1}{2^{4}}-\beta$ for all $n \in \mathbb{N}$, we obtain

$$
u_{n}=\left(\frac{69}{340}\right)^{n} u_{0} \text { and } T u_{n}=\frac{1}{5} \cdot\left(\frac{69}{340}\right)^{n} u_{0}
$$

Letting $n \rightarrow \infty$, we get that $u_{n} \rightarrow 0 \in H$ and $T u_{n} \rightarrow 0 \in H$. We notice that 0 is a fixed point of $T$ in $H$. Next, we will show that $T$ has a unique fixed point. Suppose that $u^{*}, q \in H$ are two distinct fixed points of $T$. Then,

$$
0<d_{b}\left(u^{*}, q\right)=d_{b}\left(T u^{*}, T q\right)=d_{b}\left(\frac{1}{5} u^{*}, \frac{1}{5} q\right)=\frac{1}{25} d_{b}\left(u^{*}, q\right)
$$

a contradiction. Therefore, 0 is the unique fixed point of $T$ in $H$.

Our next theorem is the Kannan type fixed point theorem for a complete convex $b$-metric space.

Theorem 2. Let $\left(H, d_{b}, w\right)$ be a complete convex b-metric space with constant $s>1$, and let the mapping $T: H \rightarrow H$ be defined as

$$
d_{b}(T u, T v) \leq k\left[d_{b}(u, T u)+d_{b}(v, T v)\right], \text { for all } u, v \in H
$$

and for some $k \in\left[0, \frac{1}{2}\right)$. Let us choose $u_{0} \in H$ in such a way that $d_{b}\left(u_{0}, T u_{0}\right)=M<\infty$ and define $u_{n}=w\left(u_{n-1}, T u_{n-1} ; \alpha_{n-1}\right)$ for $n \in \mathbb{N}$ and $\alpha_{n-1} \in\left(0, \frac{1}{4 s^{2}}\right]$. If $k \in\left[0, \frac{1}{4 s^{2}}\right]$, then $T$ has a unique fixed point in $H$.

Proof. Note that for any $n \in \mathbb{N}$, we have

$$
d_{b}\left(u_{n}, u_{n+1}\right)=d_{b}\left(u_{n}, w\left(u_{n}, T u_{n} ; \alpha_{n}\right)\right) \leq\left(1-\alpha_{n}\right) d_{b}\left(u_{n}, T u_{n}\right)
$$

and

$$
\begin{aligned}
d_{b}\left(u_{n}, T u_{n}\right)= & d_{b}\left(w\left(u_{n-1}, T u_{n-1} ; \alpha_{n-1}\right), T u_{n}\right) \\
\leq & \alpha_{n-1} d_{b}\left(u_{n-1}, T u_{n}\right)+\left(1-\alpha_{n-1}\right) d_{b}\left(T u_{n-1}, T u_{n}\right) \\
\leq & s \alpha_{n-1} d_{b}\left(u_{n-1}, T u_{n-1}\right)+s \alpha_{n-1} d_{b}\left(T u_{n-1}, T u_{n}\right) \\
& +d_{b}\left(T u_{n-1}, T u_{n}\right) \\
\leq & s \alpha_{n-1} d_{b}\left(u_{n-1}, T u_{n-1}\right) \\
& +\left(s \alpha_{n-1}+1\right) k\left[d_{b}\left(u_{n-1}, T u_{n-1}\right)+d_{b}\left(u_{n}, T u_{n}\right)\right] \\
= & \left(s \alpha_{n-1}+s \alpha_{n-1} k+k\right) d_{b}\left(u_{n-1}, T u_{n-1}\right) \\
& +\left(s \alpha_{n-1} k+k\right) d_{b}\left(u_{n}, T u_{n}\right)
\end{aligned}
$$

i.e.,

$$
\left[1-\left(s \alpha_{n-1} k+k\right)\right] d_{b}\left(u_{n}, T u_{n}\right) \leq\left(s \alpha_{n-1}+s \alpha_{n-1} k+k\right) d_{b}\left(u_{n-1}, T u_{n-1}\right)
$$

Since

$$
s \alpha_{n-1} k+k \leq\left(\frac{1}{4 s}+1\right) k<\frac{5}{4} \cdot \frac{1}{4 s^{2}}<1
$$

then

$$
d_{b}\left(u_{n}, T u_{n}\right) \leq \frac{s \alpha_{n-1}+s \alpha_{n-1} k+k}{1-\left(s \alpha_{n-1} k+k\right)} d_{b}\left(u_{n-1}, T u_{n-1}\right)
$$


Denote $\lambda_{n-1}=\frac{s \alpha_{n-1}+s \alpha_{n-1} k+k}{1-\left(s \alpha_{n-1} k+k\right)}$ for $n \in \mathbb{N}$. We deduce that

$$
\lambda_{n-1}=\frac{s \alpha_{n-1}+s \alpha_{n-1} k+k}{1-\left(s \alpha_{n-1} k+k\right)}<\frac{\frac{5}{4}}{1-\left(s \alpha_{n-1} k+k\right)}-1<\frac{\frac{5}{4}}{1-\frac{5}{4} \cdot \frac{1}{4 s^{2}}}-1<\frac{9}{11} .
$$

Combining this and inequality (5) with the assumptions of the theorem, we get

$$
d_{b}\left(u_{n}, T u_{n}\right) \leq \lambda_{n-1} d_{b}\left(u_{n-1}, T u_{n-1}\right)<\frac{9}{11} d_{b}\left(u_{n-1}, T u_{n-1}\right)
$$

which implies that $\left\{d_{b}\left(u_{n}, T u_{n}\right)\right\}$ is a decreasing sequence of non-negative reals. Hence, there exists $\gamma \geq 0$ such that

$$
\lim _{n \rightarrow \infty} d_{b}\left(u_{n}, T u_{n}\right)=\gamma
$$

We will show that $\gamma=0$. Suppose $\gamma>0$. Letting $n \rightarrow \infty$ in (6), we obtain that $\gamma \leq \frac{9}{11} \cdot \gamma<\gamma$, a contradiction. Hence, we get that $\gamma=0$; i.e.,

$$
\lim _{n \rightarrow \infty} d_{b}\left(u_{n}, T u_{n}\right)=0
$$

Moreover, by inequality (4), we obtain

$$
d_{b}\left(u_{n}, u_{n+1}\right) \leq\left(1-\alpha_{n}\right) d_{b}\left(u_{n}, T u_{n}\right)<d_{b}\left(u_{n}, T u_{n}\right)
$$

which implies that $\lim _{n \rightarrow \infty} d_{b}\left(u_{n}, u_{n+1}\right)=0$. Now we will show that $\left\{u_{n}\right\}$ is a Cauchy sequence. Indeed, if $\left\{u_{n}\right\}$ is not a Cauchy sequence, then there exist $\varepsilon_{0}>0$ and the subsequences $\left\{u_{\theta(l)}\right\}$ and $\left\{u_{\eta(l)}\right\}$ of $\left\{u_{n}\right\}$ such that $\theta(l)$ is the smallest natural index with $\theta(l)>\eta(l)>l$,

$$
d_{b}\left(u_{\theta(l)}, u_{\eta(l)}\right) \geq \varepsilon_{0}
$$

and

$$
d_{b}\left(u_{\theta(l)-1}, u_{\eta(l)}\right)<\varepsilon_{0}
$$

Then, we conclude that

$$
\varepsilon_{0} \leq d_{b}\left(u_{\theta(l)}, u_{\eta(l)}\right) \leq s\left[d_{b}\left(u_{\theta(l)}, u_{\eta(l)+1}\right)+d_{b}\left(u_{\eta(l)+1}, u_{\eta(l)}\right)\right],
$$

which implies that

$$
\frac{\varepsilon_{0}}{s} \leq \limsup _{l \rightarrow \infty} d_{b}\left(u_{\theta(l)}, u_{\eta(l)+1}\right)
$$


Noticing that

$$
\begin{aligned}
d_{b}\left(u_{\theta(l)}, u_{\eta(l)+1}\right)= & d_{b}\left(w\left(u_{\theta(l)-1}, T u_{\theta(l)-1} ; \alpha_{\theta(l)-1}\right), u_{\eta(l)+1}\right) \\
\leq & \alpha_{\theta(l)-1} d_{b}\left(u_{\theta(l)-1}, u_{\eta(l)+1}\right)+\left(1-\alpha_{\theta(l)-1}\right) d_{b}\left(T u_{\theta(l)-1}, u_{\eta(l)+1}\right) \\
\leq & \alpha_{\theta(l)-1} d_{b}\left(u_{\theta(l)-1}, u_{\eta(l)+1}\right)+\left(1-\alpha_{\theta(l)-1}\right) s\left[d_{b}\left(T u_{\theta(l)-1}, T u_{\eta(l)+1}\right)\right. \\
& \left.+d_{b}\left(T u_{\eta(l)+1}, u_{\eta(l)+1}\right)\right] \\
\leq & \alpha_{\theta(l)-1} d_{b}\left(u_{\theta(l)-1}, u_{\eta(l)+1}\right)+\left(1-\alpha_{\theta(l)-1}\right) s\left[k d_{b}\left(u_{\theta(l)-1}, T u_{\theta(l)-1}\right)\right. \\
& \left.+(k+1) d_{b}\left(T u_{\eta(l)+1}, u_{\eta(l)+1}\right)\right] \quad \text { (for some } k \in\left[0, \frac{1}{2}\right) \text { satisfying (3)) } \\
\leq & \alpha_{\theta(l)-1}\left[s d_{b}\left(u_{\theta(l)-1}, u_{\eta(l)}\right)+s d_{b}\left(u_{\eta(l)}, u_{\eta(l)+1}\right)\right] \\
& +\left(1-\alpha_{\theta(l)-1}\right) s\left[k d_{b}\left(u_{\theta(l)-1}, T u_{\theta(l)-1}\right)+(k+1) d_{b}\left(T u_{\eta(l)+1}, u_{\eta(l)+1}\right)\right],
\end{aligned}
$$

we obtain

$$
\limsup _{l \rightarrow \infty} d_{b}\left(u_{\theta(l)}, u_{\eta(l)+1}\right) \leq \frac{1}{4 s^{2}} s \cdot \varepsilon_{0}<\frac{1}{s} \varepsilon_{0}
$$

a contradiction. Thus $\left\{u_{n}\right\}$ is a Cauchy sequence in $H$. By the completeness of $H$, it follows that there exists $u^{*} \in H$ such that

$$
\lim _{n \rightarrow \infty} d_{b}\left(u_{n}, u^{*}\right)=0
$$

Now we will show that $u^{*}$ is a fixed point of T. Since

$$
\begin{aligned}
d_{b}\left(u^{*}, T u^{*}\right) & \leq s\left[d_{b}\left(u^{*}, u_{n}\right)+d_{b}\left(u_{n}, T u^{*}\right)\right] \\
& \leq s d_{b}\left(u^{*}, u_{n}\right)+s^{2}\left[d_{b}\left(u_{n}, T u_{n}\right)+d_{b}\left(T u_{n}, T u^{*}\right)\right] \\
& \leq s d_{b}\left(u^{*}, u_{n}\right)+s^{2} d_{b}\left(u_{n}, T u_{n}\right)+s^{2} k\left[d_{b}\left(u_{n}, T u_{n}\right)+d_{b}\left(u^{*}, T u^{*}\right)\right],
\end{aligned}
$$

we conclude that

$$
\begin{aligned}
\left(1-s^{2} k\right) d_{b}\left(u^{*}, T u^{*}\right) & \leq s d_{b}\left(u^{*}, u_{n}\right)+\left(s^{2}+s^{2} k\right) d_{b}\left(u_{n}, T u_{n}\right) \\
& \leq s d_{b}\left(u^{*}, u_{n}\right)+\left(s^{2}+s^{2} k\right) \cdot\left(\frac{9}{11}\right)^{n} d_{b}\left(u_{0}, T u_{0}\right) .
\end{aligned}
$$

Consequently, we get that $\lim _{n \rightarrow \infty} d_{b}\left(u^{*}, T u^{*}\right)=0$, so $u^{*}$ is a fixed point of $T$.

In order to show the uniqueness of the fixed point, suppose that $q \in H, q \neq u^{*}$, is another fixed point of $T$. Then $T q=q$. However,

$$
0<d_{b}\left(u^{*}, q\right)=d_{b}\left(T u^{*}, T q\right) \leq k d_{b}\left(u^{*}, T u^{*}\right)+k d_{b}(q, T q)=0,
$$

a contradiction. Hence, $u^{*}=q$ which completes the proof.

Next, we will give an example of applying Theorem 2.

Example 5. Let $H=\mathbb{R}^{+} \cup\{0\}$ and define the mapping $T: H \rightarrow H$ by the formula

$$
T u= \begin{cases}0, & \text { if } u \in\left[0, \frac{\sqrt{5}}{2}\right), \\ \frac{1}{4 u}, & \text { if } u \in\left[\frac{\sqrt{5}}{2},+\infty\right) .\end{cases}
$$


For any $u, v \in H$, we define $d_{b}: H \times H \rightarrow[0,+\infty)$ by the formula $d_{b}(u, v)=(u-v)^{2}$ and the mapping $w: H \times H \times[0,1] \rightarrow H$ as

$$
w(u, v ; \alpha)=\alpha u+(1-\alpha) v .
$$

Let $u_{0}$ be the initial value and $u_{n}=w\left(u_{n-1}, T u_{n-1} ; \alpha_{n-1}\right)$, where $\alpha_{n-1}=\frac{1}{4 s}$. If $k=\frac{1}{4 s^{2}}$, then $T$ has $a$ unique fixed point in $\mathrm{H}$.

Proof. From Example 4, it follows that $(H, d, w)$ is a convex $b$-metric space with $s=2$. We claim that $T$ satisfies inequality

$$
d_{b}(T u, T v) \leq k\left[d_{b}(u, T u)+d_{b}(v, T v)\right]
$$

for any $u, v \in H$. In order to prove it, we will consider the following four cases.

(i) If $u, v \in\left[0, \frac{\sqrt{5}}{2}\right)$, then it is easy to see that inequality (7) holds.

(ii) If $u \in\left[0, \frac{\sqrt{5}}{2}\right)$ and $v \in\left[\frac{\sqrt{5}}{2},+\infty\right)$, then

$$
\begin{aligned}
d_{b}(T u, T v)-\frac{1}{16}\left[d_{b}(u, T u)+d(v, T v)\right] & =\left(\frac{1}{4 v}\right)^{2}-\frac{1}{16}\left[u^{2}+\left(v-\frac{1}{4 v}\right)^{2}\right] \\
& \leq\left(\frac{1}{4 v}\right)^{2}-\frac{1}{16}\left(v-\frac{1}{4 v}\right)^{2} \leq 0
\end{aligned}
$$

which implies that

$$
d_{b}(T u, T v) \leq \frac{1}{16}\left[d_{b}(u, T u)+d_{b}(v, T v)\right]
$$

holds for any $u \in\left[0, \frac{\sqrt{5}}{2}\right)$ and $v \in\left[\frac{\sqrt{5}}{2},+\infty\right)$.

(iii) If $u \in\left[\frac{\sqrt{5}}{2},+\infty\right)$ and $v \in\left[0, \frac{\sqrt{5}}{2}\right)$, then, similarly to case (ii), we can also get that inequality (7) holds.

(iv) If $u, v \in\left[\frac{\sqrt{5}}{2},+\infty\right)$, then

$$
\begin{aligned}
d_{b}(T u, T v)-\frac{1}{16}\left[d_{b}(u, T u)+d_{b}(v, T v)\right] & =\frac{1}{16}\left(\frac{1}{u}-\frac{1}{v}\right)^{2}-\frac{1}{16}\left[\left(u-\frac{1}{4 u}\right)^{2}+\left(v-\frac{1}{4 v}\right)^{2}\right] \\
& =\frac{1}{16}\left\{\frac{15}{16}\left(\frac{1}{u^{2}}+\frac{1}{v^{2}}\right)+1-\left[\left(u^{2}+v^{2}\right)+\frac{2}{u v}\right]\right\} \\
& \leq \frac{1}{16}\left[\frac{15}{16}\left(\frac{1}{u^{2}}+\frac{1}{v^{2}}\right)+1-\left[2 u v+\frac{2}{u v}\right]\right] \\
& \leq \frac{1}{16}\left[\frac{15}{16}\left(\frac{1}{u^{2}}+\frac{1}{v^{2}}\right)+1-4\right]<0,
\end{aligned}
$$

which shows that

$$
d_{b}(T u, T v)<\frac{1}{16}\left[d_{b}(u, T u)+d_{b}(v, T v)\right]
$$

holds for all $u, v \in\left[\frac{\sqrt{5}}{2},+\infty\right)$. Summarizing, inequality (7) holds for any $u, v \in H$.

Next, we will claim that $T$ has a unique fixed point in $H$. In order to do it, we will consider the following two cases. 
(a) If $u_{0}<\frac{\sqrt{5}}{2}$, then

$$
\begin{aligned}
T u_{0} & =0 \\
u_{1} & =\frac{1}{8} u_{0}+\frac{7}{8} T u_{0}=\frac{1}{8} u_{0} \\
u_{2} & =\frac{1}{8} u_{1}+\frac{7}{8} T u_{1}=\left(\frac{1}{8}\right)^{2} u_{0} \\
u_{3} & =\frac{1}{8} u_{2}+\frac{7}{8} T u_{2}=\left(\frac{1}{8}\right)^{3} u_{0}, \\
& \cdots \\
u_{n} & =\frac{1}{8} u_{n-1}+\frac{7}{8} T u_{n-1}=\left(\frac{1}{8}\right)^{n} u_{0} .
\end{aligned}
$$

Obviously, $u_{n} \rightarrow 0$ as $n \rightarrow \infty$.

(b) If $u_{0} \geq \frac{\sqrt{5}}{2}$, then

$$
\begin{aligned}
T u_{0} & =\frac{1}{4 u_{0}} \\
u_{1} & =\frac{1}{8} u_{0}+\frac{7}{8} T u_{0}, \\
\frac{u_{1}}{u_{0}} & =\frac{1}{8}+\frac{7}{32} \frac{1}{u_{0}^{2}} \leq \frac{3}{10} .
\end{aligned}
$$

If $0 \leq u_{1}<\frac{\sqrt{5}}{2}$, then $T u_{1}=0$. From case (a), it follows that $u_{n} \rightarrow 0$ as $n \rightarrow \infty$. If $u_{1} \geq \frac{\sqrt{5}}{2}$, then $\frac{u_{2}}{u_{1}}=\frac{1}{8}+\frac{7}{32} \cdot \frac{1}{u_{1}^{2}} \leq \frac{3}{10}$. From the above procedure, we can assume that $u_{n-1} \geq \frac{\sqrt{5}}{2}$. Then, we obtain

$$
\frac{u_{n}}{u_{n-1}}=\frac{1}{8}+\frac{7}{32} \cdot \frac{1}{u_{n-1}^{2}} \leq \frac{3}{10}
$$

and

$$
\frac{u_{n}}{u_{0}}=\frac{u_{1}}{u_{0}} \cdot \frac{u_{2}}{u_{1}} \cdot \ldots \cdot \frac{u_{n}}{u_{n-1}} \leq\left(\frac{3}{10}\right)^{n},
$$

which implies that $u_{n} \leq\left(\frac{3}{10}\right)^{n} u_{0}$. Hence, $\lim _{n \rightarrow \infty} u_{n}=0$, where 0 is a fixed point of $T$. Actually, 0 is the unique fixed point of $T$ in $H$. Indeed, suppose that $q \in\left[\frac{\sqrt{5}}{2},+\infty\right)$ is also a fixed point of $T$. Then $T q=q$; that is,

$$
q=T q=\frac{1}{4 q}
$$

which implies

$$
q=\frac{1}{2}<\frac{\sqrt{5}}{2}
$$

a contradiction. Thus the proof is finished.

Next, we will consider the problem for the T-stability of Mann's iteration for the above two kinds of mappings in complete convex $b$-metric spaces. We first recall the following useful results.

Lemma 1 ([35]). Let $\left\{k_{n}\right\},\left\{l_{n}\right\}$ be non-negative sequences satisfying $k_{n+1} \leq h k_{n}+l_{n}$ for all $n \in N$, $0 \leq h<1, \lim _{n \rightarrow \infty} l_{n}=0$. Then $\lim _{n \rightarrow \infty} k_{n}=0$.

In 2008, Qing and Rhoades [24] introduced the concept of T-stability of the iteration procedure in complete metric spaces in the following: 
Definition 5 ([24]). Let $T$ be a self-map on a complete metric space $\left(H, d_{b}\right)$. Assume that $u_{n+1}=f\left(T, u_{n}\right)$ is an iteration sequence, which yields a sequence $u_{n}$ of points from $H$. Then the sequence $u_{n+1}=f\left(T, u_{n}\right)$ is said to be T-stable if $\left\{u_{n}\right\}$ converges to a fixed point $u^{*}$ of $T$, and if $\left\{v_{n}\right\}$ is a sequence in $H$ such that $\lim _{n \rightarrow \infty} d_{b}\left(v_{n+1}, f\left(T, v_{n}\right)\right)=0$, then we have $\lim _{n \rightarrow \infty} v_{n}=u^{*}$.

Now we show the notion of the weak $T$-stability of the iteration procedure.

Definition 6. Let $T$ be a self-map on a complete metric space $\left(H, d_{b}\right)$. Assume that $u_{n+1}=f\left(T, u_{n}\right)$ is an iteration sequence, which yields a sequence $u_{n}$ of points from $H$. Then the iteration procedure $u_{n+1}=f\left(T, u_{n}\right)$, is said to be weakly $T$-stable if $\left\{u_{n}\right\}$ converges to a fixed point $u^{*}$ of $T$, and if $\left\{v_{n}\right\}$ is a sequence in $H$ such that $\lim _{n \rightarrow \infty} d_{b}\left(v_{n+1}, f\left(T, v_{n}\right)\right)=0$ and sequence $\left\{d_{b}\left(v_{n}, T v_{n}\right)\right\}$ is bounded, then $\lim _{n \rightarrow \infty} v_{n}=u^{*}$.

Remark 1. It is not difficult to see that if an iteration is T-stable, then it is also weakly T-stable. However, one is not sure if the converse is not true.

Theorem 3. Under the assumptions of Theorem 1, if, additionally, $\lim _{n \rightarrow \infty} \alpha_{n}=0$, then Mann's iteration is weakly T-stable.

Proof. By virtue of Theorem 1, we deduce that $u^{*}$ is a unique fixed point of $T$ in $H$. Assume that $\left\{v_{n}\right\}$ is a sequence in $H$ which satisfies $\lim _{n \rightarrow \infty} d_{b}\left(v_{n+1}, w\left(v_{n}, T v_{n} ; \alpha_{n}\right)\right)=0$ and $\left\{d_{b}\left(v_{n}, T v_{n}\right)\right\}$ is bounded. We obtain

$$
\begin{aligned}
d_{b}\left(v_{n+1}, u^{*}\right) \leq & s\left[d_{b}\left(v_{n+1}, w\left(v_{n}, T v_{n} ; \alpha_{n}\right)\right)+d_{b}\left(w\left(v_{n}, T v_{n} ; \alpha_{n}\right), u^{*}\right)\right] \\
\leq & s d_{b}\left(v_{n+1}, w\left(v_{n}, T v_{n} ; \alpha_{n}\right)\right)+s^{2}\left[d_{b}\left(w\left(v_{n}, T v_{n} ; \alpha_{n}\right), T v_{n}\right)\right. \\
& \left.+d_{b}\left(T v_{n}, u^{*}\right)\right] \\
\leq & s d_{b}\left(v_{n+1}, w\left(v_{n}, T v_{n} ; \alpha_{n}\right)\right)+s^{2}\left[\alpha_{n} d_{b}\left(v_{n}, T v_{n}\right)+\beta d_{b}\left(v_{n}, u^{*}\right)\right] \\
= & s d_{b}\left(v_{n+1}, w\left(v_{n}, T v_{n} ; \alpha_{n}\right)\right)+s^{2} \alpha_{n} d_{b}\left(v_{n}, T v_{n}\right)+s^{2} \beta d_{b}\left(v_{n}, u^{*}\right) \\
\leq & s d_{b}\left(v_{n+1}, w\left(v_{n}, T v_{n} ; \alpha_{n}\right)\right)+s^{2} \alpha_{n} d_{b}\left(v_{n}, T v_{n}\right)+\frac{1}{s^{2}} d_{b}\left(v_{n}, u^{*}\right) .
\end{aligned}
$$

Noticing that $\frac{1}{s^{2}}<1, \lim _{n \rightarrow \infty} \alpha_{n}=0, \lim _{n \rightarrow \infty} d_{b}\left(v_{n+1}, w\left(v_{n}, T v_{n} ; \alpha_{n}\right)\right)=0$ and $\left\{d_{b}\left(v_{n}, T v_{n}\right)\right\}$ is bounded, and taking into account Lemma 1 , we get that

$$
\lim _{n \rightarrow \infty} d_{b}\left(v_{n}, u^{*}\right)=0,
$$

which completes the proof.

Theorem 4. Under all the assumptions of Theorem 2, if $\lim _{n \rightarrow \infty} \alpha_{n}=0$ and if the positive real numbers $k$ and $s$ from Theorem 2 satisfy, additionally, condition $\frac{k s^{3}}{1-k s}<1$, then Mann's iteration is weakly T-stable.

Proof. From Theorem 2, it follows that $T$ has a unique fixed point $u^{*}$ in $H$. Assume that $\left\{v_{n}\right\}$ is a sequence in $H$ which satisfies

$$
\lim _{n \rightarrow \infty} d_{b}\left(v_{n+1}, w\left(v_{n}, T v_{n} ; \alpha_{n}\right)\right)=0
$$


and $\left\{d_{b}\left(v_{n}, T v_{n}\right)\right\}$ is bounded. We obtain

$$
\begin{aligned}
d_{b}\left(v_{n+1}, u^{*}\right) \leq & s\left[d_{b}\left(v_{n+1}, w\left(v_{n}, T v_{n} ; \alpha_{n}\right)\right)+d_{b}\left(w\left(v_{n}, T v_{n} ; \alpha_{n}\right), u^{*}\right)\right] \\
\leq & s d_{b}\left(v_{n+1}, w\left(v_{n}, T v_{n} ; \alpha_{n}\right)\right)+s^{2}\left[d_{b}\left(w\left(v_{n}, T v_{n} ; \alpha_{n}\right), T v_{n}\right)\right. \\
& \left.+d_{b}\left(T v_{n}, u^{*}\right)\right] \\
\leq & s d_{b}\left(v_{n+1}, w\left(v_{n}, T v_{n} ; \alpha_{n}\right)\right)+s^{2}\left[\alpha_{n} d_{b}\left(v_{n}, T v_{n}\right)+d_{b}\left(T v_{n}, u^{*}\right)\right] .
\end{aligned}
$$

Moreover, for any $n \in \mathbb{N}$, we have

$$
\begin{aligned}
d_{b}\left(T v_{n}, u^{*}\right)=d_{b}\left(T v_{n}, T u^{*}\right) & \leq k d_{b}\left(v_{n}, T v_{n}\right) \\
& \leq k s\left[d_{b}\left(v_{n}, u^{*}\right)+d_{b}\left(T v_{n}, u^{*}\right)\right]
\end{aligned}
$$

which implies $d_{b}\left(T v_{n}, u^{*}\right) \leq \frac{k s}{1-k s} d_{b}\left(v_{n}, u^{*}\right)$. Hence,

$$
d_{b}\left(v_{n+1}, u^{*}\right) \leq \frac{k s^{3}}{1-k s} d_{b}\left(v_{n}, u^{*}\right)+s d_{b}\left(v_{n+1}, w\left(v_{n}, T v_{n} ; \alpha_{n}\right)\right)+s^{2} \alpha_{n} d_{b}\left(v_{n}, T v_{n}\right) .
$$

Noticing that $\frac{k s^{3}}{1-k s}<1, \lim _{n \rightarrow \infty} \alpha_{n}=0, \lim _{n \rightarrow \infty} d_{b}\left(v_{n+1}, w\left(v_{n}, T v_{n} ; \alpha_{n}\right)\right)=0$ and $\left\{d_{b}\left(v_{n}, T v_{n}\right)\right\}$ is bounded, and by virtue of Lemma 1 , we get that $\lim _{n \rightarrow \infty} d_{b}\left(v_{n}, u^{*}\right)=0$.

\section{Applications}

In this section we apply Theorem 1 in order to show the existence and uniqueness of the solution of the Fredholm linear integral equation:

$$
u(t)=f(t)+\lambda \int_{a}^{b} K(t, \tau) u(\tau) d \tau .
$$

Theorem 5. Consider the linear integral Equation (8) with the continuous function $K(t, \tau)$, where $a \leq t, \tau \leq b$ and $f \in H[a, b]$. Let $M=\max _{a \leq t, \tau \leq b}|K(t, \tau)|$ and $m>2$ be an arbitrary real number. If $|\lambda|<\frac{1}{2 m M(b-a)}$, then the linear integral Equation (8) has a unique solution on the interval $[a, b]$. Moreover, the solution is exhibited as follows:

$$
u(t)=f(t)+\lambda \lim _{n \rightarrow \infty} \int_{a}^{b} K(t, \tau) u_{n}(\tau) d \tau,
$$

where $u_{0}(t)=u_{0}, \alpha_{n-1}=\frac{1}{16}-\frac{1}{4 m^{2}}$ and

$$
u_{n}(t)=\alpha_{n-1} u_{n-1}(t)+\left(1-\alpha_{n-1}\right)\left[f(t)+\lambda \int_{a}^{b} K(t, \tau) u_{n-1}(\tau) d \tau\right], n=1,2,3, \cdots .
$$

Proof. Let $H=C[a, b]$ and define $d_{b}: H \times H \rightarrow[0,+\infty)$ by the formula

$$
d_{b}(u, v)=\max _{a \leq t, \tau \leq b}|u(t)-v(t)|^{2} .
$$

Define a self-map $T$ on $H$ by

$$
T u(t)=f(t)+\lambda \int_{a}^{b} K(t, \tau) u(\tau) d \tau, \text { for all } u \in H[a, b] .
$$

Set

$$
u_{n}=w\left(u_{n-1}, T u_{n-1} ; \alpha_{n-1}\right)=\alpha_{n-1} u_{n-1}+\left(1-\alpha_{n-1}\right) T u_{n-1},
$$


where $\alpha_{n-1}=\frac{1}{16}-\frac{1}{4 m^{2}}$. It is obvious that $\left(H, d_{b}, w\right)$ is a complete convex $b$-metric space with $s=2$. Combining (10) and (11), we get

$$
\begin{aligned}
d_{b}(T u, T v) & =\max _{a \leq t, \tau \leq b}|T u(t)-T v(t)|^{2} \\
& =\max _{a \leq t, \tau \leq b}\left|\lambda \int_{a}^{b} K(t, \tau) u(\tau)-K(t, \tau) v(\tau) d \tau\right|^{2} \\
& \leq \max _{a \leq t, \tau \leq b}|\lambda|^{2}\left|\int_{a}^{b}\right| K(t, \tau)|| u(\tau)-v(\tau)|d \tau|^{2} \\
& \leq[M(b-a)|\lambda|]^{2} \max _{a \leq t, \tau \leq b}|u(\tau)-v(\tau)|^{2} \\
& =[M(b-a)|\lambda|]^{2} d_{b}(u, v)<\frac{1}{4 m^{2}} d_{b}(u, v) .
\end{aligned}
$$

Hence, $T$ is a contraction mapping on $H\left(\beta=\frac{1}{4 m^{2}}\right)$. Meanwhile, by Theorem $1, T$ has a unique fixed point $u(t) \in H$ satisfying $\lim _{n \rightarrow \infty} u_{n}(t)=u(t)$ and $T u(t)=u(t)$, which means that $u(t)$ is the solution of (8). Now we will show that

$$
\int_{a}^{b} K(t, \tau) u(\tau) d \tau=\lim _{n \rightarrow \infty} \int_{a}^{b} K(t, \tau) u_{n}(\tau) d \tau
$$

Indeed,

$$
\begin{aligned}
\limsup _{n \rightarrow \infty} \int_{a}^{b} K(t, \tau)\left(u_{n}(\tau)-u(\tau)\right) d \tau & \leq \limsup _{n \rightarrow \infty} \int_{a}^{b}|K(t, \tau)|\left|u_{n}(\tau)-u(\tau)\right| d \tau \\
& \leq M(b-a) \limsup _{n \rightarrow \infty} \max \left|u_{n}(\tau)-u(\tau)\right| .
\end{aligned}
$$

Since

$$
\lim _{n \rightarrow \infty} d\left(u_{n}(t), u(t)\right)=\lim _{n \rightarrow \infty} \max \left(u_{n}(t)-u(t)\right)^{2}=0,
$$

it is not difficult to see that

$$
\lim _{n \rightarrow \infty} \int_{a}^{b} K(t, \tau)\left(u_{n}(\tau)-u(\tau)\right) d \tau=0 .
$$

Thus, Equation (9) holds.

Author Contributions: Conceptualization, L.C.; Investigation, C.L. and R.K.; Writing—review and editing, Y.Z. All authors have read and agreed to the published version of the manuscript.

Funding: This research was funded by the Training Program for Youth Innovation Talents of Heilongjiang Educational Committee under grant UNPYSCT-2017078, the Postdoctoral Science Foundation of Heilongjiang Province under grant LBH-Q18067 and the Introduction and Cultivation Project of Young and Innovative Talents in Universities of Shandong Province.

Acknowledgments: The authors would like to thank the referees for valuable comments and suggestions which helped to improve this paper.

Conflicts of Interest: The authors declare no conflict of interest.

\section{References}

1. Ran, A.C.M.; Reurings, M.C.B. A fixed point theorem in partially ordered sets and some applications to matrix equations. Proc. Am. Math. Soc. 2004, 132, 1435-1443. [CrossRef]

2. Wang, H.Q.; Song, X.H.; Chen, L.L.; Xie, W. A secret sharing scheme for quantum gray and color images based on encryption. Int. J. Theor. Phys. 2019, 58, 1626-1650. [CrossRef]

3. Banach, S. Sur les operations dans les ensembles abstraits et leur application aux equations integrales. Fundam. Math. 1922, 3, 133-181. [CrossRef] 
4. Chen, L.; Gao, L.; Chen, D. Fixed point theorems of mean nonexpansive set-valued mappings in Banach spaces. J. Fixed Point Theory Appl. 2017, 19, 2129-2143. [CrossRef]

5. Chen, L.; Gao, L.; Zhao, Y. A new iterative scheme for finding attractive points of $(\alpha, \beta)$-generalized hybrid set-valued mappings. J. Nonlinear Sci. Appl. 2017, 10, 1228-1237. [CrossRef]

6. Chen, L.; Zou, J.; Zhao, Y.; Zhang, M. Iterative approximation of common attractive points of $(\alpha, \beta)$-generalized hybrid set-valued mappings. J. Fixed Point Theory Appl. 2019, 21, 58. [CrossRef]

7. Mihet, D. A Banach contraction theorem in fuzzy metric spaces. Fuzzy Sets Syst. 2004, 144, 431-439. [CrossRef]

8. Ruzhansky, M.; Cho, Y.J.; Agarwal, P.; Area, I. Advances in Real and Complex Analysis with Applications; Springer: London, UK, 2017.

9. Sawangsup, K.; Sintunavarat, W.; Cho, Y.J. Fixed point theorems for orthogonal F-contraction mappings on O-complete metric spaces. J. Fixed Point Theory Appl. 2020, 22, 10. [CrossRef]

10. Saipara, P.; Kumam, P.; Cho, Y.J. Random fixed point theorems for Hardy-Rogers self-random operators with applications to random integral equations. Stochastics 2018, 90, 297-311. [CrossRef]

11. Reich, S. Fixed points of contractive functions. Boll. Della Unione Mat. Ital. 1972, 5, $26-42$.

12. Reich, S. Kannan's fixed point theorem. Boll. Unione Mat. Ital. 1971, 4, 1-11.

13. Mongkolkeha, C.; Cho, Y.J.; Kumam, P. Fixed point theorems for simulation functions in b-metric spaces via the wt-distance. Appl. Gen. Topol. 2017, 18, 91-105. [CrossRef]

14. Cho, Y.J.; Rassias, T.M.; Saadati, R. Fuzzy Operator Theory in Mathematical Analysis; Springer: London, UK, 2018.

15. Aghajani, A.; Abbas, M.; Roshan, J.R. Common fixed point of generalized weak contractive mappings in partially ordered $b$-metric spaces. Math. Slovaca 2014, 64, 941-960. [CrossRef]

16. Reich, S. Nonlinear evolution equations and nonlinear ergodic theorems. Nonlinear-Anal. Theory Methods Appl. 1977, 1, 319-330. [CrossRef]

17. Reich, S.; Zaslavski, A.J. Genericity in Nonlinear Analysis; Springer: London, UK, 2014.

18. Czerwik, S. Contraction mappings in b-metric spaces. Acta Math. Inform. Univ. Ostrav. 1993, 1, 5-11.

19. Boriceanu, M.; Bota, M.; Petruşel, A. Multivalued fractals in b-metric spaces. Open Math. 2010, 8, 367-377. [CrossRef]

20. Boriceanu, M.; Petrusel, A.; Rus, I. Fixed point theorems for some multivalued generalized contractions in b-metric spaces. Int. J. Math. Stat. 2010, 6, 65-76.

21. Bota, M.F.; Karapınar, E. A note on "Some results on multi-valued weakly Jungck mappings in $b$-metric space". Open Math. 2013, 11, 1711-1712. [CrossRef]

22. Tiammee, J.; Suantai, S.; Cho, Y.J. Existence theorems of a new set-valued MT-contraction in $b$-metric spaces endowed with graphs and applications. Fixed Point Theory 2018, 19, 785-800. [CrossRef]

23. Yamaod, O.; Sintunavarat, W.; Cho, Y.J. Existence of a common solution for a system of nonlinear integral equations via fixed point methods in $b$-metric spaces. Open Math. 2016, 14, 128-145. [CrossRef]

24. Qing, Y.; Rhoades, B. T-stability of picard iteration in metric spaces. Fixed Point Theory Appl. 2008, 2008, 418971. [CrossRef]

25. Todorčević, V. Harmonic Quasiconformal Mappings and Hyperbolic Type Metrics; Springer: London, UK, 2019.

26. Aleksić, S.; Došenović, T.; Mitrović, Z.D.; Radenović, S. Remarks on common fixed point results for generalized $\alpha_{*}-\psi$-contraction multivalued mappings in b-metric spaces. Adv. Fixed Point Theory 2019, 9,1-16.

27. Todorčević, V. Subharmonic behavior and quasiconformal mappings. Anal. Math. Phys. 2019, 9, 1211-1225. [CrossRef]

28. Chen, L.; Liu, X.; Zhao, Y. Exponential stability of a class of nonlinear systems via fixed point theory. Nonlinear Anal. 2020, 196, 111784. [CrossRef]

29. Takahashi, W. A convexity in metric space and nonexpansive mappings. I. Kodai Math. Semin. Rep. 1970, 22, 142-149. [CrossRef]

30. Goebel, K.; Kirk, W.A. Iteration processes for nonexpansive mappings. Contemp. Math. 1983, 21, 115-123.

31. Ding, X.P. Iteration processes for nonlinear mappings in convex metric spaces. J. Math. Anal. Appl. 1988, 132, 114-122. [CrossRef]

32. Reich, S.; Shafrir, I. Nonexpansive iterations in hyperbolic spaces. Nonlinear Anal. 1990, 19, 537-558. [CrossRef] 
33. Reich, S. Weak convergence theorems for nonexpansive mappings in Banach spaces. J. Math. Anal. Appl. 1979, 67, 274-276. [CrossRef]

34. Borwein, J.; Reich, S.; Shafrir, I. Krasnoselskii-Mann iterations in normed spaces. Can. Math. Bull. 1992, 35, 21-28. [CrossRef]

35. Liu, Q. The convergence theorems of the sequence of Ishikawa iterates for hemicontractive mappings. J. Math. Anal. Appl. 1990, 146, 301-305.

(C) 2020 by the authors. Licensee MDPI, Basel, Switzerland. This article is an open access article distributed under the terms and conditions of the Creative Commons Attribution (CC BY) license (http:/ / creativecommons.org/licenses/by/4.0/). 